\title{
Pixel-wise parameter adaptation for single-exposure extension of the image dynamic range
}

\author{
R. Carmona-Galán \\ Inst. Microelectrónica de \\ Sevilla (IMSE-CNM), \\ CSIC-Univ. Sevilla (Spain) \\ Tel. +34954466666 \\ rcarmona@imse- \\ cnm.csic.es
}

\author{
J. A. Leñero-Bardallo \\ Inst. Microelectrónica de \\ Sevilla (IMSE-CNM), \\ CSIC-Univ. Sevilla (Spain) \\ Tel. +34954466666 \\ juanle@imse- \\ cnm.csic.es
}

\author{
J. Fernández Berni \\ Inst. Microelectrónica de \\ Sevilla (IMSE-CNM), \\ CSIC-Univ. Sevilla (Spain) \\ Tel. +34954466666 \\ berni@imse- \\ cnm.csic.es
}

\author{
Á. Rodríguez-Vázquez \\ Inst. Microelectrónica de \\ Sevilla (IMSE-CNM), \\ CSIC-Univ. Sevilla (Spain) \\ Tel. +34 954466666 \\ angel@imse- \\ cnm.csic.es
}

\begin{abstract}
High dynamic range imaging is central in application fields like surveillance, intelligent transportation and advanced driving assistance systems. In some scenarios, methods for dynamic range extension based on multiple captures have shown limitations in apprehending the dynamics of the scene. Artifacts appear that can put at risk the correct segmentation of objects in the image. We have developed several techniques for the on-chip implementation of single-exposure extension of the dynamic range. We work on the upper extreme of the range, i. e. administering the available full-well capacity. Parameters are adapted pixel-wise in order to accommodate a high intra-scene range of illuminations.
\end{abstract}

\section{CCS Concepts}

- Hardware $\rightarrow$ Communication hardware, interfaces and storage $\rightarrow$ Displays and imagers $\cdot$ Computing methodologies $\rightarrow$ Computer vision $\rightarrow$ Image and video acquisition.

\section{Keywords}

High-dynamic range imaging; adaptive image capture.

\section{INTRODUCTION}

The ability of capturing a wide range of illumination conditions within the same frame is much wanted in application fields without controlled lighting conditions [1]. Surveillance, assisted driving, intelligent transportation, etc., are developed in scenarios in which high intra-scene ranges of illumination can be expected [2]. Even in industrial applications - the domain of machine vision-, activities like laser welding or melted glass molding, generate highly illuminated areas. Without the appropriate dynamic range to allocate these diverse illumination values, obtaining a detailed view of the brightest zones can easily obscure other elements in the scene.

The dynamic range (DR) of an image sensor is the range of illumination conditions from which the sensor is still able to extract meaningful information simultaneously. In particular, we are referring to the intra-scene dynamic range, i. e. the ratio between the brightest and the darkest image intensities that can be distinguished within the same frame. Extension of the dynamic range can be done at the two extremes. Increasing light sensitivity and reducing noise allows for dynamic range extension beyond its lower limit [3]. In this paper we will take care of the upper limit of DR. The target of these techniques will be to make the sensor able to capture information from pixels that, in principle, were illuminated beyond their saturation threshold. That is, of course, without losing information from the less illuminated areas.
The most popular techniques for high dynamic range imaging can be classified into three different groups [1]. There are methods that use a companding response of the sensor [4], others employ multiple image captures [5], and others work on the evaluation of the saturation condition at pixel level to locally modify pixel parameters like the integration time or the voltage range [6].

Our approach falls into this last category. On the one hand, trusting on the nonlinear operation of the sensor has several drawbacks like the increased influence of mismatch between pixels, difficulties in cancelling the spatial noise, and a limited voltage range that renders a loss of image contrast [1]. On the other hand, multiple captures with misaligned integration times can generate inexistent edges and distort the interpretation of the scene [7].

The two chips reviewed in this paper [8] [9] work with a single exposure. They evaluate the illumination conditions locally and concurrently with image acquisition, and they have the necessary elements to correct pixel parameters to accommodate a wider intra-scene DR. In [8] this evaluation allows for the local adaptation of the integration time. In [9] the voltage range is extended as needed by the asynchronous tagging of saturation events at each pixel. As a consequence, in both examples the image is ready after exposure without post-processing.

In the next section, we will describe the basic principles for dynamic range extension. Then we will explain that saturation conditions can be assessed without having to wait to the end of exposure, allowing for concurrent adaptation of the pixel parameters. After that, we will review the characteristics of a sensor chip that adapts the integration time of each pixel to the local illumination average [8]. Then, a prototype sensor whose pixels self-reset after asynchronously tagging the saturation events is described [9]. Common principles of both approaches and advantages over other dynamic range extension methods will be highlighted in the final discussion and the conclusions.

\section{LIMITS OF THE DYNAMIC RANGE}

The most common method for image sensing in CMOS is a technology compatible photodiode, e. g. n-well/p-subs, in integrating mode (Fig. 1). In the presence of an illumination source that generates an irradiance, $E(\lambda)$, which is a function of $\lambda$, on the surface of the diode, a photocurrent is prompted:

$$
I_{p h}=\frac{q A_{D}}{h c} \int_{\lambda_{-}}^{\lambda_{+}} \eta(\lambda) E(\lambda) d \lambda
$$


where $\eta(\lambda)$ is the quantum efficiency, $A_{D}$ is the diode area, and the integral is evaluated along all wavelengths that contribute to irradiate the diode. The pixel voltage $V_{p h}$ is built upon the sensing capacitance, $C_{s}$, which in this case is the reverse-biased diode capacitance. In the case of a $4 \mathrm{~T}$ architecture, i. e. a pixel with a pinned photodiode, the sensing capacitance corresponds to the floating diffusion. After being reset to a value $V_{\mathrm{RST}}$, the photocurrent $I_{\mathrm{ph}}$ discharges the node $V_{p h}$ during the exposure time $t_{\text {exp }}$ :

$$
V_{p h}=V_{\mathrm{RST}}-\frac{I_{p h} t_{\mathrm{exp}}}{C_{s}}
$$

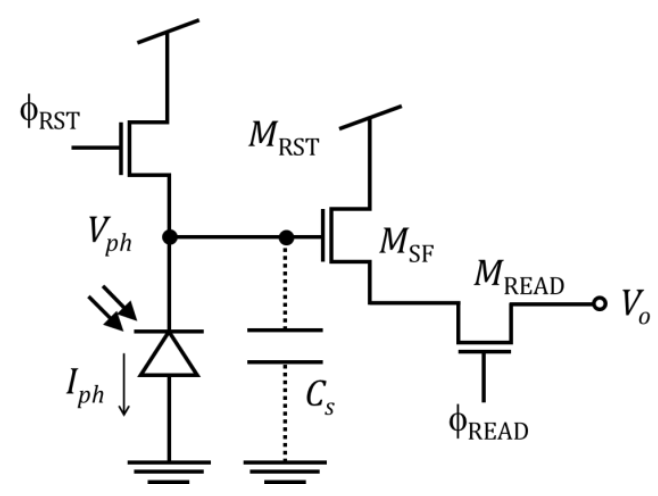

Fig. 1. 3T active pixel sensor showing diode capacitance

If the photocurrent is considered to be constant along the complete integration period, the amount of charge extracted from $C_{S}$ over time is given by:

$$
Q(t)=C_{s}\left[V_{\mathrm{RST}}-V_{p h}(t)\right]=I_{p h} t
$$

being $t=0$ the time instant in which the photocurrent integration has begun. The lowest illumination signal that can be detected, that corresponds to photocurrent $I_{\text {min }}$, is determined from the noise floor, $Q_{\text {noise}}$, which is obtained by accounting for all the contributions referred to the input node:

$$
I_{\min }=Q_{\text {noise }} / t_{\text {exp }}
$$

Notice that the longer the exposure the smaller the light intensities that can be detected. Ideally, for a $t_{\exp } \rightarrow \infty$, the minimum detectable signal will approach zero, if it was not for $1 / f$-noise.

On the other extreme, the highest light intensity that can be sensed, which correspond to the maximum photocurrent $I_{\max }$, is given by the maximum amount of charge that can be extracted from the sensing capacitance for the given voltage range:

$$
I_{\max }=C_{s}\left(V_{\mathrm{RST}}-V_{\min }\right) / t_{\exp }
$$

where $V_{\min }$ is the lowest value that can be reached by $V_{p h}$. This charge corresponds to the full-well capacity in electrons multiplied by the elementary charge. Beyond that, the photodiode is not able to extract more charge from $C_{s}$, and it is said that the pixel has reached saturation.

The dynamic range of a single pixel is defined by the ratio between the maximum and the minimum detectable signals:

$$
\mathrm{DR}=20 \log _{10}\left(\frac{I_{\max }}{I_{\min }}\right)
$$

From Eq. (3) it can be seen that reducing the noise level, $Q_{\text {noise }}$, allows for detecting a fainter light signal, i. e. a smaller $I_{\text {min }}$. This represents an extension of the lower limit of DR without affecting the upper limit. It requires a careful design of the readout channels, and can easily involve an intervention on the chip fabrication technology.

In order to act on the upper limit of DR, i. e. to be able to sense a larger $I_{\max }, \mathrm{C}_{s}$ or the voltage range $\left(V_{\max }-V_{\min }\right)$ need to be increased. In both cases, the noise floor can eventually rise. On the one hand, a larger capacitance results in reduced pixel sensitivity, i. e. more electrons are required to raise the voltage to detectable levels, and therefore $I_{\min }$ increases as well. On the other hand a larger voltage range introduces higher quantization noise unless the equivalent resolution of the ADC is increased accordingly.

Another option is to modify the integration time. It is clear from Eqs. (3), (4) and (5) that simply changing $t_{\text {exp }}$ for the whole array does not have any incidence on the dynamic range. Rising $t_{\exp }$ for the same noise level, $Q_{\text {noise }}$, allows detecting a smaller $I_{\text {min }}$, but $I_{\max }$ decreases as well, thus ending is the same DR. On the contrary, a smaller $t_{\exp }$ results in higher $I_{\min }$ and $I_{\max }$ simultaneously. Again, DR does not change. A different thing will be to assign different integration times for the different pixels, what can result in a larger image dynamic range while the DR does not change pixel-wise.

\section{EXTENSION OF THE UPPER LIMIT}

Let us consider the ratio $\alpha$ between the actual photocurrent of a pixel $I_{p h}$ and the maximum current $I_{\max }$ that can be sensed for a particular exposure time $t_{\text {exp }}$. Making use of Eqs. (2) and (4) it can be said that:

$$
\alpha=\frac{I_{p h}}{I_{\max }}=\left(\frac{t_{\mathrm{exp}}}{t}\right)\left[\frac{V_{\mathrm{RST}}-V_{p h}(t)}{V_{\mathrm{RST}}-V_{\min }}\right]
$$

at any time instant during integration. Notice the important fact that $\alpha$ can be computed at any time within the exposure interval. As long as the photocurrent remains constant during the integration, it does not have to finish to anticipate if a pixel is going to be overexposed. Therefore, mechanisms can be devised to check this condition on-line to render a single-exposure extension of the dynamic range, as we will see later.

Going back to Eq. (6), as long as $\alpha \leq 1$, there is no overexposure. $V_{p h}\left(t_{\text {exp }}\right)$ will be above $V_{\text {min }}$, so the response to this particular illumination can be accommodated into the allocated voltage range. On the contrary, if $\alpha>1$, some modification of the pixel parameters is required or Eq. (6) will not hold, what means that the pixel will saturate.

At this point, one of the options is reducing the exposure time for that particular pixel. The pixel will be assigned a different exposure time, $t_{\text {exp }}^{\prime}$ so as to have $t_{\text {exp }}^{\prime}<t_{\exp }$ and $V_{p h}\left(t_{\text {exp }}^{\prime}\right) \geq$ $V_{\min }$. In these conditions, photocurrents above $I_{\max }$ will be still accommodated in the available voltage range. Of course, having a particular integration time for each pixel will represent a distortion of the relative differences between them, but we will see later how this can be attenuated to still provide all the meaningful details from the scene.

Another option, that will keep all the exposure times the same, is providing a larger voltage range, i. e. defining a $V_{\text {min }}^{\prime}<V_{\min }$ in order to accommodate larger excursions of $V_{p h}$. This is equivalent to increase the amount of charge that can be extracted from $C_{s}$, though, in order not to affect sensitivity, we need to find a mechanism for replenishing the full well capacity without changing the sensing capacitance. 


\section{ADAPTATION OF THE EXPOSURE 4.1 DR extension principle}

As already mentioned, locally modifying the integration time will allow accommodating larger illuminations. For a particular pixel, changing the exposure time from $t_{\exp }$ to $t_{\text {exp }}^{\prime}=t_{\exp } / \alpha$, with $\alpha>1$, allows to detect photocurrents as high as $\alpha I_{\max }$, for the same voltage range. The highest light intensity that can be sensed now corresponds to photocurrent:

$$
I_{\text {max }}^{\prime}=\left(\frac{t_{\text {exp }}}{t_{\text {exp }}^{\prime}}\right) I_{\text {max }}
$$

what represents an extension of the dynamic range given by:

$$
\mathrm{DR}^{\prime}-\mathrm{DR}=20 \log _{10}\left(\frac{t_{\text {exp }}}{t_{\text {exp }}^{\prime}}\right)
$$

In order to evaluate $\alpha$ at the beginning of the exposure, $t_{\mathrm{s}}$ seconds can be employed to sample $V_{p h}$ and then distribute the remaining time, $t_{\exp }-t_{\mathrm{s}}$, according to an appropriate criterion.

\subsection{Local integration time selection}

Consider then that during the sampling period, $t_{\mathrm{s}}$, all the values of the pixels are sensed in order to evaluate its corresponding $\alpha$. The selection of $t_{\mathrm{s}}$ will be arbitrary for now, we will see later that an optimum $t_{\mathrm{s}}$ for each image. Of course, $t_{\mathrm{s}}<t_{\mathrm{exp}}$. The average voltage also evolves during this interval, until reaching this point:

$$
\overline{V_{p h}}\left(t_{s}\right)=V_{\mathrm{RST}}-\frac{\overline{I_{p h}} t_{s}}{C_{s}}
$$

Let us assume that $t_{\mathrm{s}}$ has been selected so that this voltage does not progress below the midpoint of the range. Now, let us define an auxiliary per pixel reference value that starts from $\overline{V_{p h}}\left(t_{s}\right)$ and then decays according to the local photocurrent:

$$
V_{\text {ref }}(t)=V_{\mathrm{RST}}-\frac{\overline{I_{p h}}}{C_{s}} t_{s}-\frac{I_{p h}}{C_{s}}\left(t-t_{s}\right) \quad \forall t \geq t_{s}
$$

This reference voltage will help us establish the local exposure, $t_{\text {exp }}^{\prime}$. Notice that half of the pixels will have a photocurrent above $I_{p h}$ and the other half below. So we will need to allocate their integration times in the interval $\left[t_{\mathrm{s}}, t_{\mathrm{exp}}\right]$. Let us consider that the local exposure will be determined by the time instant in which $V_{\text {ref }}$ crosses the middle of the range, that is:

$$
V_{\text {ref }}\left(t_{\text {exp }}^{\prime}\right)=\frac{V_{\mathrm{RST}}+V_{\mathrm{min}}}{2}
$$

what renders:

$$
t^{\prime}{ }_{\exp }=C_{s} \frac{V_{\mathrm{RST}}-V_{\min }}{2 I_{p h}}-\frac{\overline{I_{p h}}-I_{p h}}{I_{p h}} t_{s}
$$

This means that the final voltage of the pixel, at $t_{\text {exp }}^{\prime}$, is:

$$
V_{p h}\left(t_{\text {exp }}^{\prime}\right)=\frac{V_{\mathrm{RST}}+V_{\min }}{2}+\frac{\overline{I_{p h}}-I_{p h}}{C_{s}} t_{s}
$$

Notice that for a pixel having just the average photocurrent, the final voltage will be the midpoint of the range. Pixels illuminated above the average will end in final voltages below the midpoint, and those with photocurrents below the average will end in voltages above it.
The minimum integration time that is available is $t_{\mathrm{s}}$, therefore the maximum detectable current $I_{\text {max }}^{\prime}$ is given by:

$$
I_{\text {max }}^{\prime}=\left(\frac{t_{\mathrm{exp}}}{t_{\mathrm{s}}}\right) I_{\max }
$$

The lowest detectable photocurrent is still given by Eq. (3) as it is determined by noise and the highest exposure time, which is $t_{\text {exp }}$. The dynamic range of the complete sensor is now given by:

$$
\mathrm{DR}^{\prime}=20 \log _{10}\left[\frac{t_{\mathrm{exp}}}{t_{\mathrm{s}}} \cdot \frac{C_{s}\left(V_{\mathrm{RST}}-V_{\mathrm{min}}\right)}{Q_{\text {noise }}}\right]
$$

\subsection{Dual-diode pixel implementation}

In order to implement the required sampling of the local pixel voltage and the average voltage of the array, the circuit in Fig. 2 is proposed [10].

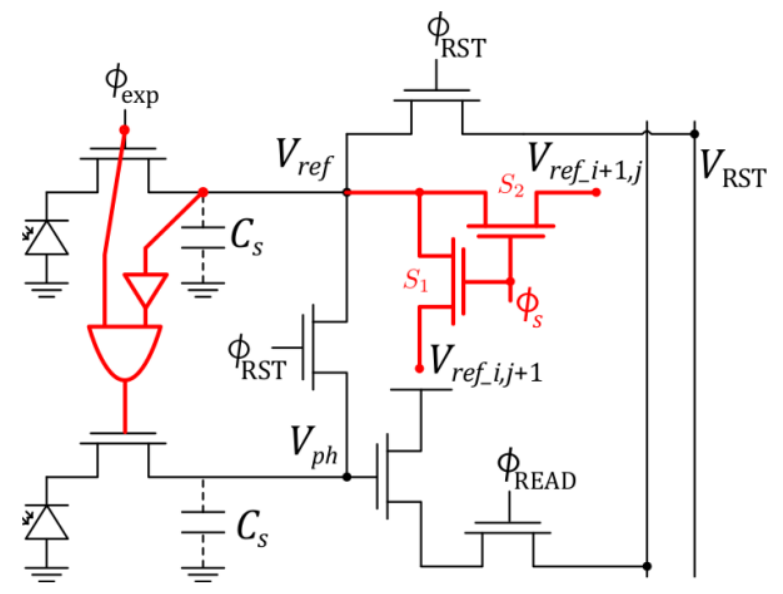

Fig. 2. Dual-diode pixel sensor

The pixel value itself is represented by $V_{p h}$, as before. Now, there is an additional photodiode, which is responsible of building voltage $V_{\text {ref }}$. This second photodiode per pixel has been employed in industry for dual concurrent exposure [11], with a large photodiode for high sensitivity and a small one for low sensitivity. In our case, the second photodiode is a non-imageforming device. It is employed to enable adaptation to global and local illumination conditions. The voltage $V_{\text {ref }}$ encodes the signal adjusting the local integration time. By making use of switches $S_{1}$ and $S_{2}$, all the pixels are connected as long as $\phi_{s}$ is on, i. e. from $t=0$ to $t=t_{s}$. The result is that, before $t_{s}$, node $V_{\text {ref }}$ represents the average voltage value of all the pixels in the array. The temporal dynamics of $V_{\text {ref }}$ are then governed by Eq. (10).

The local integration time, $t_{\text {exp }}^{\prime}$, is determined by the time it takes for $V_{r e f}$ to reach the midpoint of the voltage range, as defined by Eq. (11). In this circuit, this is implemented by the digital buffer connected to node $V_{\text {ref }}$. Its threshold voltage has been designed to coincide with the middle of the range. Therefore, the transmission gate of the other photodiode will be on until $t^{\prime}{ }_{\text {exp }}$, which is defined by Eq. (12).

This approach has been proved to work inside a region of interest (ROI) in [8]. Within a ROI, correlation between light intensities is usually high. In scenes with a high DR, we can find pixels that are much brighter or much darker than the average. In this chip, the selection of $t_{\mathrm{s}}$ permits to balance the contribution of the global and local illumination conditions to the adaptation of the pixel parameters, i. e. the integration time in this case. 


\subsection{Optimum $t_{s}$ and experimental results}

According to Eq. (15), the smaller $t_{\mathrm{s}}$ the wider the range of light intensities that can be mapped into the available voltage range. This means that the smaller $t_{\mathrm{s}}$, the less pixels will reach saturation. But on the other hand, compression of the illumination range should not be at the expense of a loss of detail. Therefore, this adaptation mechanism has been benchmarked attending not only to the number of saturated pixels, which has to be maintained at acceptable levels, but also attending to the correlation found in the image histogram. The higher the correlation, the more equalized the strengths of the different tones, and therefore the highest contrast can be appreciated.

In these conditions, there is an optimum $t_{\mathrm{s}}$ rendering the best histogram equalization while keeping a low count of saturated pixels. The optimum $t_{\mathrm{s}}$ is different for each image, so it can be subject of subsequent adaptation by recursive algorithms. Fig. 3 displays a capture of a scene with a high DR (102dB), with a prototype chip implementing this mechanism [10] using an optimized $t_{\mathrm{s}}$. Despite a spatial resolution of only $320 \times 240$ pixels, details can be appreciated both outside of the window and in the picture on the wall inside the room.

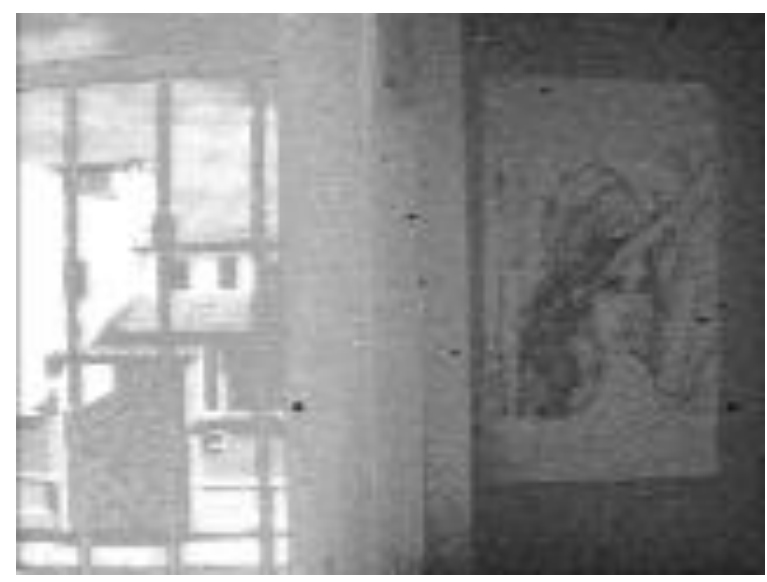

Fig. 3. Balanced image captured by a prototype chip

For a $t_{\mathrm{s}}$ below the optimum, we will be allocating some voltage range for illumination levels that do not exist in the scene. For $t_{\mathrm{s}}$ 's above the optimum, we are losing information in the form of saturated pixels.

This prototype sensor chip has been fabricated in a standard 0.18um CMOS process, and has been conceived as a vision sensor, not an image sensor. This means that the processing capabilities at the sensor plane were given priority over image quality. A better design of the sensor interface and a better process in what concerns noise will certainly render a higher DR.

\section{VOLTAGE RANGE EXTENSION}

\subsection{Self-resetting active pixel sensor}

Going back to Eq. (6), allowing $V_{p h}$ to progress below $V_{\min }$ increases the full well capacity and therefore the available dynamic range. The major difficulty to implement this extension is that the voltage range is limited in the technology. The only way to do this is to bring back the pixel to the reset state as soon as it has reached saturation, i. e. $V_{p h}(t)=V_{\min }$. 0 illustrates the waveform described by the pixel voltage in this scheme. The complete voltage range available will depend on the number of saturation events we will be able to count. Let it be $k$, the maximum voltage range is:

$$
\Delta \mathrm{V}_{p h}=\left(V_{\mathrm{RST}}-V_{\min }\right) k
$$

and the maximum detectable photocurrent is thus:

$$
I_{\text {max }}^{\prime}=k I_{\max }
$$

which identifies with an $\alpha \geq 1 \quad \forall \alpha \in \mathbb{N}^{*}$. This represents an extension of:

$$
\mathrm{DR}^{\prime}-\mathrm{DR}=20 \log _{10} k
$$

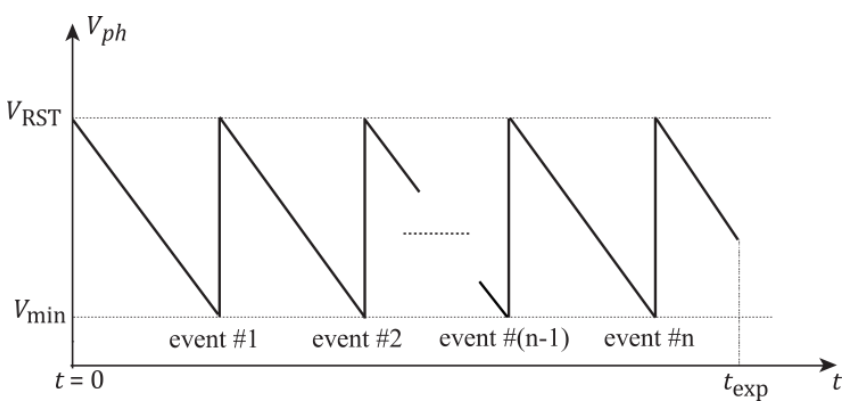

Fig. 4. Pixel voltage in self-resetting pixel

Similar approaches have been previously reported [12]. The mayor difficulty is not in the implementation of the self-reset but in the account of saturation/reset events. Storing the saturation events count within the pixel [13] certainly limits the achievable $k$, as memory needs silicon area, thus establishing a trade-off between spatial resolution and pixel voltage range. On the other hand, counting these saturation events outside of the pixel represents a synchronicity problem, i. e. there are no means to seamlessly anticipate the order in which the pixels are going to saturate. Our approach is to asynchronously tag the saturation events outside of the array, using an address-event encoding.

\subsection{Pixel architecture}

Pixel schematics are displayed in Fig. 5. Apart from the corresponding readout circuitry, consisting on a copy of the pixel voltage $V_{p h}^{\prime}$ and a source follower, the pixel contains a comparator to fix the minimum voltage $V_{\min }$ and drive the selfreset transistor $\mathrm{M}_{\mathrm{p} 3}$. This feedback loop constitute an oscillator that goes back to $V_{\mathrm{RST}}$ each time that $V_{p h}=V_{\text {min }}$. In addition to this, the self-reset pulse activates the asynchronous circuitry that handles the event communication. Knowing the number of times that a pixel has spiked and the final value of $V_{\text {out }}$, it is possible to build a digital word which value is proportional to the pixel illumination. Let us call $b$ to the number of bits that encode the analog voltage of the pixel, i. e. $V_{\text {out }}$ will be quantized into $2^{b}$ levels. Let $m$ be the number of bits that encode the maximum number of saturation events per pixel that can be tagged, $k$. Then $k=2^{m}$. The output of the chip will be encoded by a digital word of $n$ bits being:

$$
n=m+b
$$

The minimum photocurrent current that can be measured, taking into account the $\mathrm{ADC}$, is:

$$
I_{\text {min }}^{\prime}=\frac{C_{S}\left(V_{\mathrm{RST}}-V_{\mathrm{min}}\right)}{2^{b} t_{\mathrm{exp}}}
$$

which should match the limit imposed by the noise floor Eq. (3), in order not to introduce additional limitations. Besides, the maximum photocurrent that can be detected is given by: 


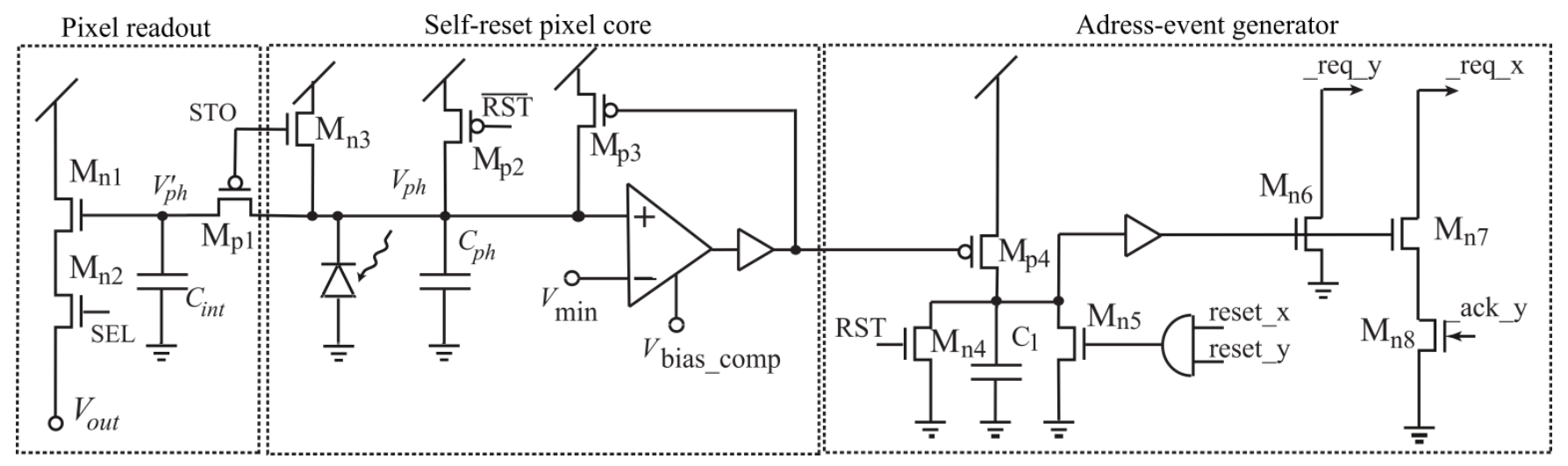

Fig. 5. Schematics of the self-resetting pixel with address-event generation for the asynchronous tagging of saturation events

$$
I_{\max }^{\prime}=2^{m} C_{S}\left(V_{\mathrm{RST}}-V_{\min }\right) / t_{\mathrm{exp}}
$$

Hence the total dynamic range achievable is now:

$$
\mathrm{DR}^{\prime}=20 \log _{10}\left(\frac{I_{\text {max }}^{\prime}}{I_{\text {min }}^{\prime}}\right)=6.02(m+b) \mathrm{dB}
$$

Limitations to this method are in the oscillation frequency of the self-resetting core and the capacity of the address-event management. For a delay loop given by:

$$
\tau_{\text {loop }}=\frac{C_{s}\left(V_{\mathrm{RST}}-V_{\text {min }}\right)}{I_{\text {max }}^{\prime}}+\frac{1}{\mathrm{BW}} \approx \frac{t_{\mathrm{exp}}}{k}
$$

the term 1/BW represents the dynamics of the amplifier, which can be designed to be negligible compared to the first term. Using Eq. (21), the time it takes for the maximum photocurrent to go over the complete voltage range $k$ times is one $k$-th of $t_{\text {exp }}$. Consider than each handshaking cycle to complete the correct tagging of a saturation event takes $t_{h}$ seconds. If it has to be done $k$ times it will take $k t_{h}$. In the most extreme case, all the $M \times N$ pixels of the image will be firing the oscillator at the highest rate, what means that:

$$
t_{h}<\frac{t_{\mathrm{exp}}}{k M N}
$$

Conversely, given the restrictions associated to the handling of events outside of the array [14], the inverse of $t_{h}$ renders a maximum number of events per second (eps), let us call it $E_{\max }$, achievable by a particular implementation of the event-tagging circuit. In this chip, for instance, event rates up to 20Meps for pixels of different rows and up to 2Meps for pixels in the same row can be handled. From Eq. (24) a limit on the achievable frame rate, $\left(1 / t_{\exp }\right)$ is found:

$$
\frac{1}{t_{\text {exp }}}<\frac{E_{\max }}{k M N}
$$

\subsection{Experimental results}

Fig. 6 displays the experimental setup and a snapshot of the graphical user interface (GUI). We have designed a custom PCB and 3D-printed a lens holder to test the sensor. An Opal Kelly XC7K160T board is attached to the test chip PCB. Also a FPGA Mezzanine Card for interfacing. In order to debug the sensor and display images in real-time, a custom interface was programmed in C++. Fig. 6 (b) shows the GUI displaying an image acquired by the chip. Several sliders to control parameters like the integration time and the analog circuit biasing have been added.
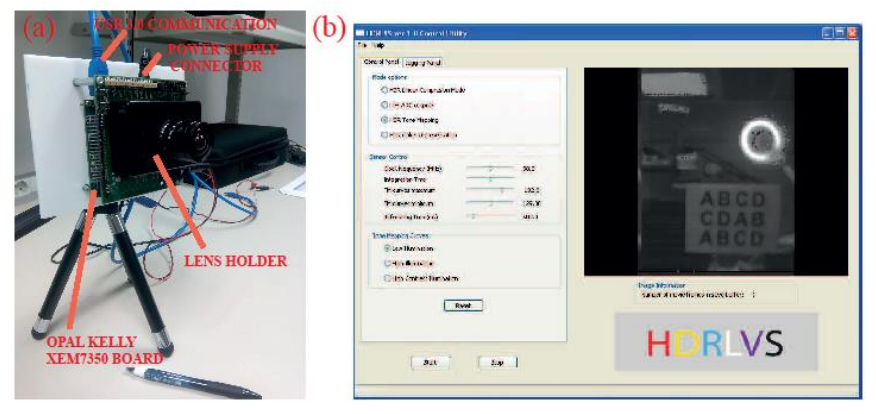

Fig. 6. Experimental setup and custom GUI for sensor debugging and data representation

Fig. 7 displays some image data capture by the chip. In the first place, picture (a) displays a HDR image of a natural scene taken with a BQ Aquaris E 4.5 smartphone operating in HDR mode. A snapshot of the same visual scene was taken with our sensor. In Fig. 7 (b) the intensity levels encoded with the sensor have been represented using a thermal code. The intra-scene dynamic range of the scene was $107 \mathrm{~dB}$. In the common ambient conditions of the laboratory, it is challenging to find scenes with an intra-scene dynamic range higher than $120 \mathrm{~dB}$ [15]. For this reason, for the preliminary test of our system, we assigned $m=12$. Thus, according to Eq. (22), if $b=8$, then $n=24$ and the maximum intra-scene dynamic range that can be measured is $120 \mathrm{~dB}$.

We have set a value of $t_{\text {exp }}=110 \mathrm{~ms}$ to capture the image. With a thermal code it is possible to assign up to 24 bits to the intensity levels representation. This representation is lineal and preserves all the scene illumination values measured with the camera. However, it is difficult to visually appreciate image details in the darkest areas. Fig. 7 (c) displays the same scene after processing the sensor's output with a tone mapping algorithm [16]. Tone mapping relates the input intensity levels with 256 greyscale levels so they can be represented in a conventional display. There is a nonlinear relation between the input intensity and grey level assigned to each pixel. Histogram equalization can be realized on the basis of the already captured pixel values. As an example, Fig. 8 (a) displays the histogram of the picture in Fig. 7. Notice that most of the information is in the lowest intensity levels, while a 
few pixels are well above them. The result with a common image sensor adapted to the average lighting is a complete loss of detail in the brightest pixels. Fig. 8 displays the appropriate tone map to accommodate the complete image within 256 greyscale levels without losing detail of neither the brightest nor the darkest areas.

The user can define curves that adapt to the image histograms to achieve more precision encoding grey levels with the illumination values that are more frequent in the visual scene. Fig. 7 (d) displays the image histograms. Fig. 7 (e) shows the tone mapping curve used the render the image of Fig. 7 (c).

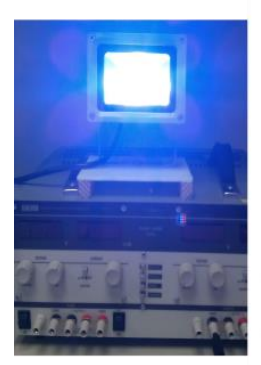

(a)

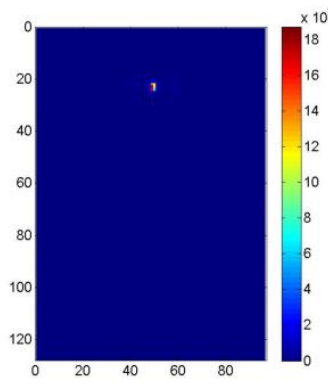

(b)

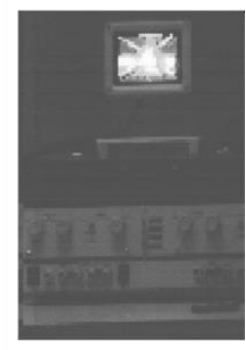

(c)
Fig. 7. Samples images: taken with a smartphone in HDR mode (a), snapshot of the same visual scene taken with our sensor displayed with a thermal code (b) and tone mapped (c)

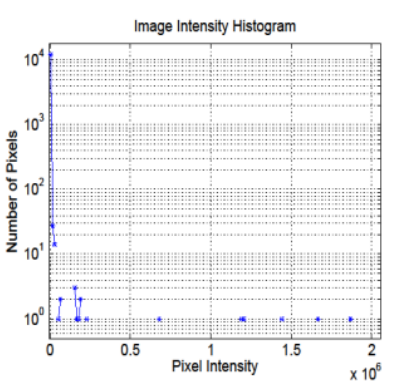

(a)

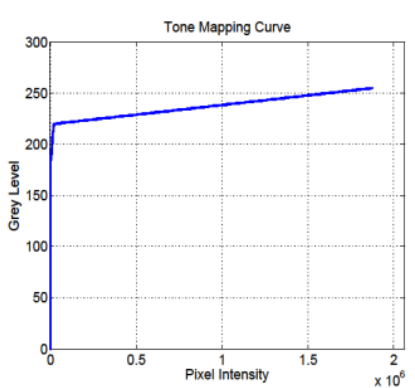

(b)
Fig. 8. Original image histogram and tone mapping curve

\section{CONCLUSIONS}

A new sensor with high dynamic operation and linear response has been presented. The sensor pixels reset themselves and never overflow during the integration time. Events are sent out every time that a pixel resets itself. The sensor can achieve high dynamic operation detecting images with intra-scene dynamic range beyond $120 \mathrm{~dB}$. The maximum high dynamic range is limited by the maximum event rate that the arbitration system can handle and by the number of bits dedicated to store the number of events associated to each pixel. Power consumption is $13 \mathrm{~mA}$ under average operation conditions. Preliminary experimental results and sample images are provided.

\section{ACKNOWLEDGMENTS}

This work has been funded by the Spanish Ministry of Economy (MINECO) through projects TEC2015-66878-C3-1-R MINECO (co-funded by the European Regional Development Fund, ERDF/FEDER), IPT-2011-1625-430000, IPC- 20111009 CDTI (also co-funded by ERDF/FEDER), Junta de Andalucía's project P12-TIC 2338 CEICE, and the Office of Naval Research (USA) through grant No. N000141410355.

\section{REFERENCES}

[1] A. Spivak et al. 2009. Wide-dynamic-range CMOS image sensors-comparative performance analysis. IEEE Trans. Electron Devices, 56, 11 (2009) 2446-2461.

[2] J. Solhusvik et al. 2013. A comparison of high dynamic range CIS technologies for automotive applications. International Image Sensor Workshop, (Snowbird Resort, Utah, USA, June 12-16, 2013).

[3] K. Mizobuchi et al. 2008. A low-noise wide dynamic range CMOS image sensor with low and high temperature resistance. Proc. SPIE, 6816 (2008) 681.64 1-8.

[4] W. F. Chou et al. 2014. A Linear-Logarithmic CMOS Image Sensor With Pixel-FPN Reduction and Tunable Response Curve. IEEE Sensors Journal, 14, 5 (2014) 1625-1632.

[5] O. Yadid-Pecht and E. R. Fossum, 1997. Wide intrascene dynamic range CMOS APS using dual sampling. IEEE Transactions on Electron Devices, 44, 10 (Oct. 1997) 17211723.

[6] X. Qian et al. 2013. An Adaptive Integration Time CMOS Image Sensor With Multiple Readout Channels. IEEE Sensors Journal, 13, 12 (2013) 4931-4939.

[7] J. Solhusvik, 2015. High Dynamic Range Capture Technologies. 1st Workshop on Advancements in Circuits and Imaging (University of Oxford, UK. September 28-29, 2015).

[8] J. Fernández-Berni et al. 2015. Real-time single-exposure ROI-driven HDR adaptation based on focal-plane reconfiguration. Proc. of SPIE, 9400, (2015) 94000K.1-6.

[9] Juan A. Leñero-Bardallo et al. A high dynamic range linear vision sensor with event asynchronous and frame-based synchronous operation. IS\&T Int. Symp. on Electronic Imaging (San Francisco, USA, February 2016).

[10] J. Fernández-Berni et al., 2016. Single-Exposure HDR Technique Based on Tunable Balance Between Local and Global Adaptation. IEEE Trans. CAS-II 63, 5 (2016) 488492.

[11] T. Willansen et al. 2015. A 1280x1080 4.2um Split-diode Pixel HDR Sensor in $110 \mathrm{~nm}$ BSI CMOS Process. International Image Sensor Workshop (Vaals, The Netherlands, June 8-11, 2015).

[12] C. Posch, D. Matolin, and R. Wohlgenannt, 2010. A QVGA $143 \mathrm{~dB}$ dynamic range asynchronous address-event PWM dynamic image sensor with lossless pixel-level video compression, IEEE Journal of Solid State Circuits, 46, 1 (2010) 259-275.

[13] T. Yamada et al., 2008. A 140dB-dynamic range MOS image sensor with in-pixel multiple-exposure synthesis. IEEE International Solid-State Circuits Conference, (San Francisco, CA, Feb. 2008) 50-594.

[14] P. Häfliger, A spike based learning rule and its implementation in analog hardware. Ph.D. dissertation (ETH Zürich, Switzerland, 2000).

[15] A. Darmont, 2008. Methods to extend the dynamic range of snapshot active pixel sensors. Proceedings of SPIE 6816, (Feb. 2008).

[16] L. Meylan, (2006). Tone mapping for high dynamic range images. Ph.D. dissertation (Ecole Polytechnique Federale de Lausane, Switzerland, 2006). 